\title{
A Novel Accident Prevention System using VANET and Remedial System using IoT
}

\author{
Prabhakar Manage \\ Assistant Prof., EC Department \\ KLE Dr.M.S.Sheshgiri College of \\ Engineering \& Technology \\ Belagavi, India
}

\author{
Vaishnavi Patil \\ EC Department \\ KLE Dr.M.S.Sheshgiri College of \\ Engineering \& Technology \\ Belagavi, India
}

\author{
Shrusti Aribenchi \\ EC Department \\ KLE Dr.M.S.Sheshgiri College of \\ Engineering \& Technology \\ Belagavi, India
}

\author{
Sahana Navalgatti \\ EC Department \\ KLE Dr.M.S.Sheshgiri College of \\ Engineering and Technology \\ Belagavi, India
}

\author{
Pranali Gomanache \\ EC Department \\ KLE Dr.M.S.Sheshgiri College of \\ Engineering and Technology \\ Belagavi, India
}

\begin{abstract}
Road accidents are human misfortune. They involve major human and financial suffering as there is no immediate help provided to the injured people. A lot of people witness the accidents but fail to offer help as they are hesitant of police investigation and allegations, and thus victims die due to lack of medical help. Presently there are various emanate technologies like Adaptive cruise control (ACS), Anti-lock braking system (ABS), Airbag system which are available only in high end cars that prevents accidents but they do not provide any remedies if accidents take place. To overcome the limitations of existing system this paper presents a solution which includes both preventive as well as remedial system. VANET technology is used in the preventive system. The remedial system focuses on providing details of the location to the nearby hospitals so that immediate help can be provided, the Internet of Things (IoT) can be used to accomplish this task. The system improves road safety, security, communication medium, performance monitoring and increases productivity.
\end{abstract}

\section{Keywords-Prevention, Remedial, VANET, IoT, Andriod}

\section{INTRODUCTION}

Increasing population has led to rapid increase in usage of vehicles which in turn is responsible for increasing death rate. WHO launched a Global Status Report on road safety which highlights that the annual road traffic death toll has reached 1.35 million per year with highest road casualty rates in developing countries [1]. Road accidents have become mundane over the past few years and it is predicted to increase by $2 \%$ every year [2]. Across 199 countries, India ranks first in the number of road accident deaths followed by China and US, the biggest reason being over speeding, drunk driving, negligence of the driver etc. Approximately $69.6 \%$ of the road accident victims fall in the age group of $18-45$ years.

The World Health Statistics 2008 stated that in Global Status Report on Road Safety states that Road Traffic Injuries were the $9^{\text {th }}$ leading cause for death and at current rates by 2030 are expected to be the $5^{\text {th }}$ leading cause of death [3] and [4]. Moreover, survey shows that each minute that an injured crash victim does not receive emergency medical care can make a large difference in their survival rate, e.g., analysis shows that reducing accident response time by one minute correlates to a six percent difference in the number of lives saved .An effective approach for reducing traffic fatalities, therefore, is to reduce the time between when an accident occurs and when first responders, such as medical personnel, are dispatched to the scene of the accident.

In order to prevent the accidents, the possibility of an emergency situation has to be predicted in advance which is a tedious task. Some preventive technologies already exists in high end cars like Intelligent head light control (BMW), Adaptive cruise control (ACC), Automatic emergency braking system etc.. With the context to Intelligent Transport System, collection of data from the vehicles is preferred by many applications [5] and [6]. To predict the accidents in advance, communication should be established between vehicles, which can be accomplished by using VANET technology. The preventive system uses VANET technologyVehicular Ad-Hoc Network where communication between vehicles takes place using Li-Fi technology. The development, research and deployment of Vehicular Ad-Hoc (VANET) technologies gives the concept of how the V2V and V2I communications improves the safety on roads [7].

The ideal situation for any accident is that the police officials and the ambulance arrive to the accident spot immediately, but this luxury is not offered to the victims in reality, because the spectators panic due to the further consequences and therefore often do not report the accidents[8]. Also if an accident takes place in a remote place there is no help provided to the injured and hence the response time for detection is high. The Internet of Things (IoT) can be used to provide notification and response to the situation automatically. The Internet of Things (IoT) is the term used to point out the communication between people to things and things to things. Thus the remedial system proposed in this research work detects the accident location and updates the pivotal information like location of the accident, victim details to the hospital web page. 


\section{EXPERIEMENTAL SETUP}

\section{A. PREVENTIVE SYSTEM:}

The system is providing a measure in order to prevent the accidents by implementing the VANET technology. The VANET or Vehicular Ad-Hoc Network is a technology that considers moving cars as nodes in a network in order to create a mobile network. This technology turns every participating car into a wireless router or node, allowing cars within the range of 100-300 meters to connect and communicate and in turn create a network with a wide rage. In layman terms it is a communication between vehicle to vehicle (V2V) or vehicle to infrastructure (V2I) [9].

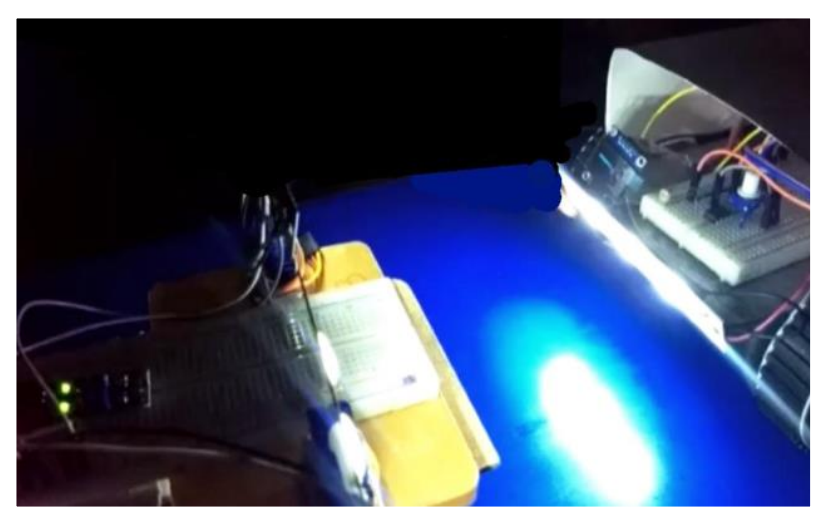

Fig 1. Experimental set up to implement VANET technology

The idea involves implementing VANET technology using the Li-Fi concept. Li-Fi is a wireless communication technology which makes use of light to transmit data between the devices. The main aim of this idea is to establish communication between the vehicles. The communication involves sending and receiving of messages (which may include data regarding the speed, intensity etc.) between the vehicles in binary format

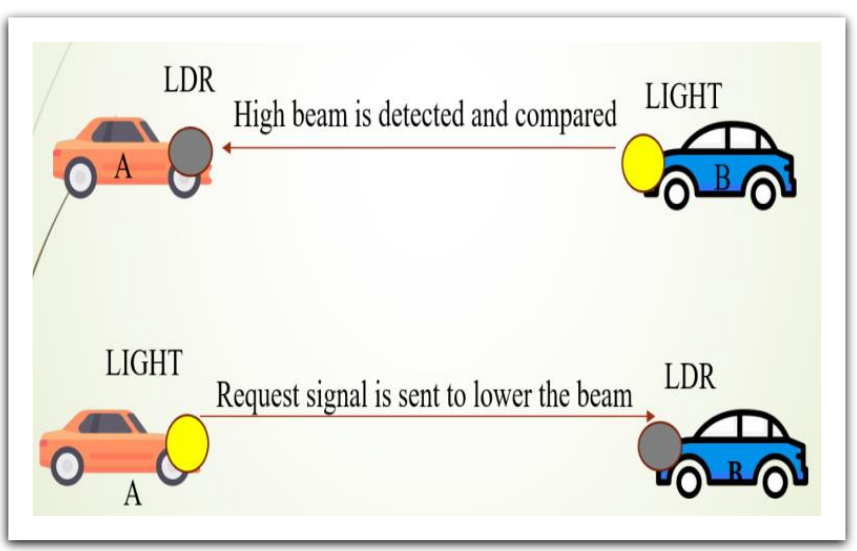

Fig 2. Preventive System block diagram

Consider a scenario where in Car A and Car B move in opposite direction and are about to collide at a point (fig 2). Car B is moving with a high intensity beam while Car A is moving with the standard beam in opposite direction to that of Car B. The high intensity of Car B falls on the LDR (Light Dependent Resister) module placed in Car A. The comparator placed in Car A then compares it with the standard threshold value, if the result is higher than that of the standard threshold then Car A sends a signal to lower the beam to Car B in binary format through light. Car B has another LDR and comparator, the LDR receives the light signal while the comparator decodes it as a result of which the intensity of the beam is reduced automatically. All the processing and controlling operations are performed by a microcontroller which is coded accordingly. Thus accidents can be prevented.

\section{B.REMEDIALSYSTEM}

In case the accident takes place the purpose of the remedial system will be to provide immediate help to the victim with the help of IoT technology.

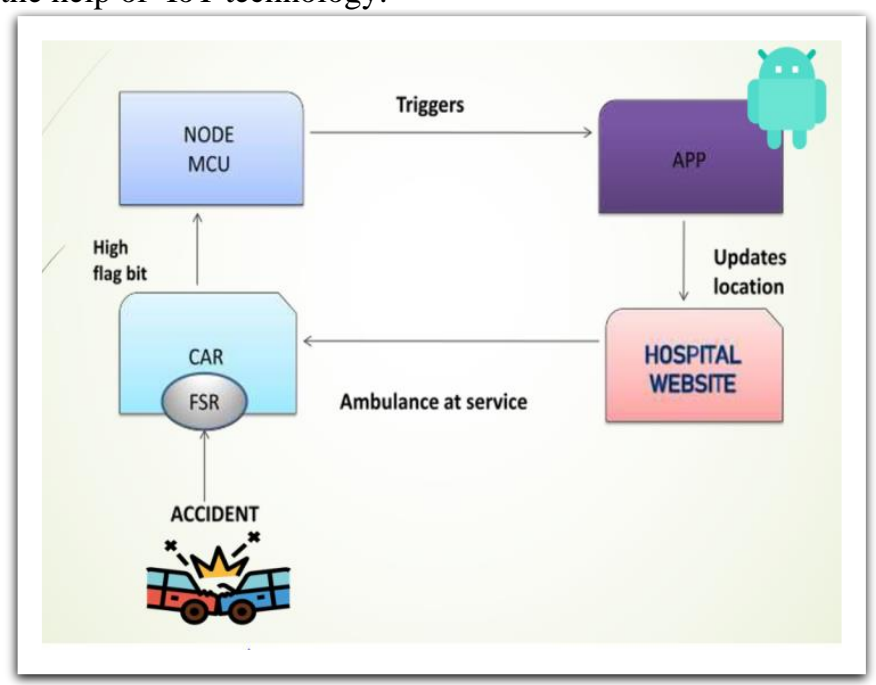

Fig. 3 Block diagram of the remedial system

Fig 3 shows the block diagram of remedial system. When there is a collision between two vehicles there is an impact on the sensor, to check whether this impact is high enough to have caused an accident ,this value is compared with standard value which is already been fed in the controller. If the impact value is greater than that of the standard threshold value then the controller triggers the node MCU which is a processor and Wi-Fi module. Each node MCU has its own IP address, this IP address is fed in the android app which will have to be installed before using this system. The app then fetches the current location from the phone and then sends this location along with the personal information( fed in the app while singing up) to the hospital webpage and the ambulance is sent from the nearest hospital

For this system to work an android app has to be installed. The user has to register first by providing all the personal details like name, address, emergency contact, blood group. The user then has to login with email id and registered password. The arrival of the ambulance will be notified in the app along with some car information as indicated in Fig 4. 


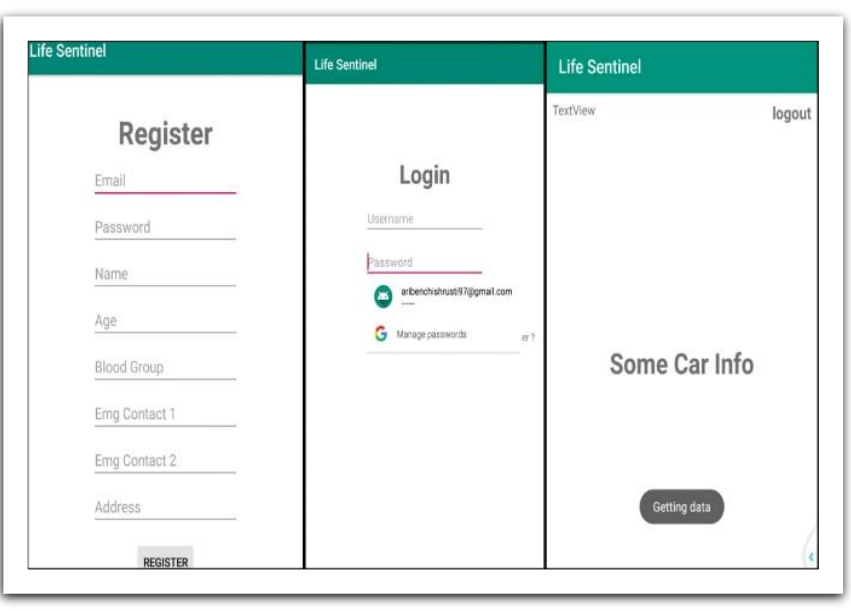

Fig. 4 Process to login into the app

The remedial system includes a web page which has to be merged with the hospital web page (Fig. 5). The accident location and victim details are updated so that the hospital can send an ambulance to the accident site to provide the required help. Furthermore, the accident location can also be sent to the emergency contact using a GSM module [10].

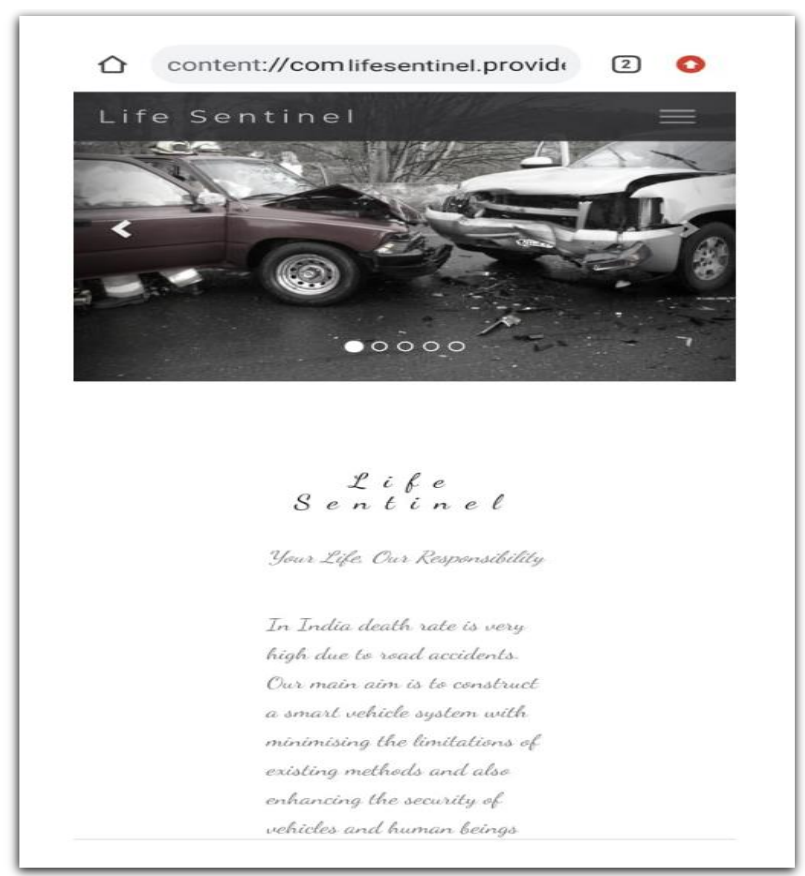

Fig 5. Hospital web page

The receptionist monitoring the webpage will get the information about all the different accident locations. So the receptionist will have to choose the nearest accident site to serve the aid for victim, once the request is confirmed from the web page, the location disappears so that other hospital doesn't serve the same patient and thereby increases the efficiency(Fig 5).

\section{RESULTS}

The main objective of the preventive system is to establish communication between the vehicles. So when two cars are moving in opposite direction the one with a high intensity beam will receive a request message ( the request message is as shown in the Fig 6 ) to lower the intensity of the light and thus lowering the intensity of light will be done automatically.

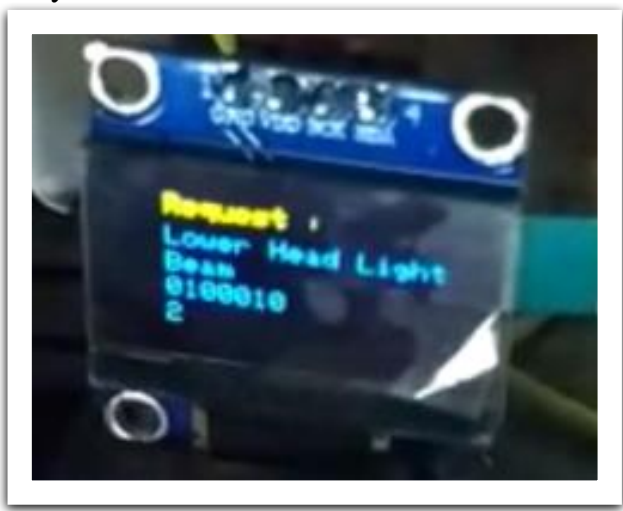

Fig. 6 Showing the result of the preventive system to lower the headlight The main purpose of the remedial system is to provide immediate help to the victim, this is achieved by updating the accident location in the hospital webpage (as shown in Fig 7) so that the nearby hospital can send an ambulance to the location to serve the aid and also this acknowledgement is provided in the android app.

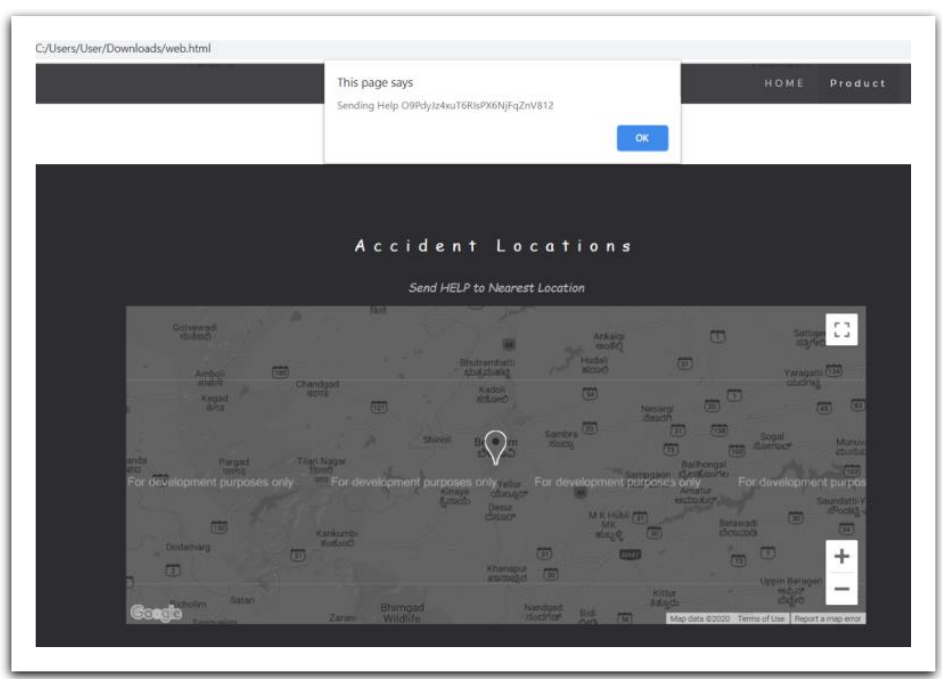

Fig. 7 Showing the accident location in the hospital web page

Along with the accident location the victim details are also updated in the web page to provide the necessary help to the victims as soon as possible ( as shown in figure 8)

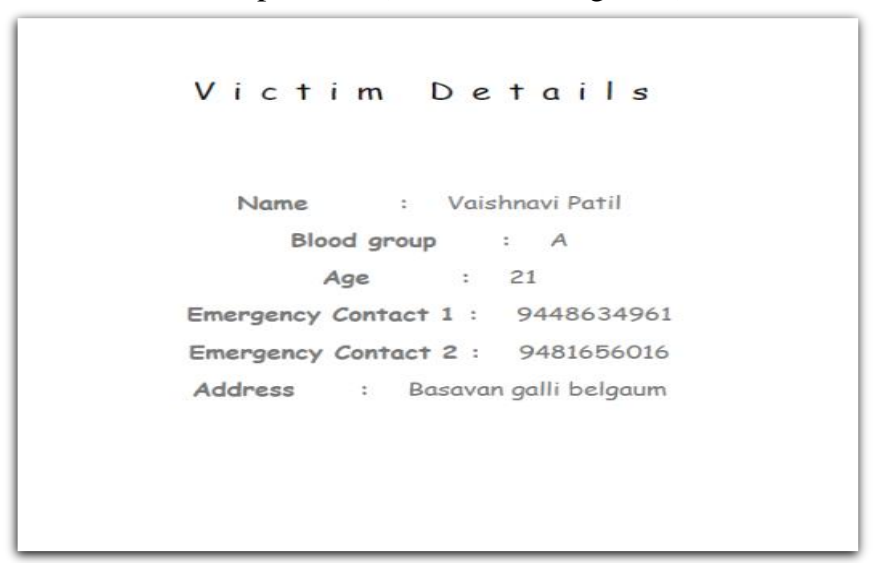

Fig. 8 Showing the victim details that are updated in the hospital web page 


\section{CONCLUSION}

The preventive system will enable communication between the vehicles by implementing VANET technology using the Li-Fi concept which will prevent the collisions of vehicles that may occur due to various reasons, thus the proposed system alerts the authoritative driver by predicting an emergency situation in advance.

Also if an accident takes place, post collision the impact detected by the sensor triggers the Node MCU. When this happens the accident location along with the biometrics details, emergency contacts etc. is updated in the web page. The hospital nearby to the accident location can serve the request thus providing security to the vehicle and the driver.

\section{REFERENCES}

[1] Nanda, Sayanee \& Joshi, Harshada \& Khairnar, Smita. (2018). "An IOT Based Smart System for Accident Prevention and Detection." pp. 1-6. Doi: 10.1109/ICCUBEA.2018.8697663.

[2] P. Krishnan, "Design of Collision Detection System for Smart Car Using Li-Fi and Ultrasonic Sensor," in IEEE Transactions on Vehicular Technology, vol. 67, no. 12, pp. 11420-11426, Dec. 2018

[3] B. S. Anil, K. A. Vilas and S. R. Jagtap, "Intelligent system for vehicular accident detection and notification," 2014 International Conference on Communication and Signal Processing, Melmaruvathur, 2014, pp. 1238-1240. Doi:10.1109/ICCSP.2014.6950048.

[4] "Road Accidents In India 2010" Government Of India Ministry Of Road Transport And Highways Transport Research Wing New Delhi December 2011,pp. 1-53

[5] J.Zhao, Haitao \& Yu, Hongsu \& Li, Dapeng \& Mao, Tianqi \& Zhu, Hongbo. (2019). Vehicle Accident Risk Prediction based on AdaBoostSO in VANETs. IEEE Access. PP. 1-1. 10.1109/ACCESS.2019.2894176

[6] Turcanu I, Klingler F, Sommer C, et al. "Duplicate Suppression for Efficient Floating Car Data Collection in Heterogeneous LTE-DSRC Vehicular Networks," Computer Communications, vol. 123, pp.54- 64, Jun. 2018.

[7] S. I. Fadilah, A. R. M. Shariff and M. N. M. Hilmi, "Crash Avoidance Based Periodic Safety Message Dissemination Protocol for Vehicular Ad Hoc Network," 2019 IEEE 89th Vehicular Technology Conference (VTC2019-Spring), Kuala Lumpur, Malaysia, 2019, pp. 1-7.

[8] Ali and M. Eid, "An automated system for Accident Detection," 2015 IEEE International Instrumentation and Measurement Technology Conference (I2MTC) Proceedings, Pisa, 2015, pp. 16081612.Doi:10.1109/I2MTC.2015.7151519

[9] Fadilah, S. I., \& Shariff, A. R. M. (2014, January). A time gap interval for safe following distance (TGFD) in avoiding car collision in wireless vehicular networks (VANET) envi-ronment. In Intelligent Systems, Modelling and Simulation (ISMS), 2014 5th International Conference on (pp. 683-689). IEEE.

[10]A. Shaik et al., "Smart Car: An IoT Based Accident Detection System," 2018 IEEE Global Conference on Internet of Things (GCIoT), Alexandria, Egypt, 2018, pp. 1-5. 